LA W RENCE LIVERM ORE NATIONAL LABORATORY

Collective Dissipation from

Saddle to Scission in a

Microscopic Approach

W. Younes, D. Gogny

October 1, 2012 
This document was prepared as an account of work sponsored by an agency of the United States government. Neither the United States government nor Lawrence Livermore National Security, LLC, nor any of their employees makes any warranty, expressed or implied, or assumes any legal liability or responsibility for the accuracy, completeness, or usefulness of any information, apparatus, product, or process disclosed, or represents that its use would not infringe privately owned rights. Reference herein to any specific commercial product, process, or service by trade name, trademark, manufacturer, or otherwise does not necessarily constitute or imply its endorsement, recommendation, or favoring by the United States government or Lawrence Livermore National Security, LLC. The views and opinions of authors expressed herein do not necessarily state or reflect those of the United States government or Lawrence Livermore National Security, LLC, and shall not be used for advertising or product endorsement purposes.

This work performed under the auspices of the U.S. Department of Energy by Lawrence Livermore National Laboratory under Contract DE-AC52-07NA27344. 


\title{
Collective dissipation from saddle to scission in a microscopic approach
}

\author{
W. Younes and D. Gogny
}

(Dated: September 28, 2012)

\begin{abstract}
We calculate the energy dissipated into collective modes during the descent from saddle to scission for the most likely fission mode of ${ }^{240} \mathrm{Pu}$, with respect to the quadrupole and octupole degrees of freedom. The energy dissipated in fission directly impacts calculations of the spectrum of particles emitted by the fragments. The results presented here rely on the Bohr approximation to the timedependent generator-coordinate method in order to describe the evolution of the fissioning system to scission. A comparison of the normalized flux in a two-dimensional calculation, with respect to the quadrupole and octupole modes, and a one-dimensional calculation along the most likely fission path is used to deduce the energy dissipated into transverse collective modes. A value of $3.4 \pm 0.1 \mathrm{MeV}$ is obtained for the collective energy dissipation.
\end{abstract}




\section{INTRODUCTION}

One of the outstanding questions in nuclear fission is what fraction of the energy gained in the descent from saddle to scission is converted to pre-scission kinetic energy, and what fraction is dissipated into excitation energy of the fragments. An accurate accounting of this energy partition is essential to the proper calculation of fission-fragment properties $[1,7]$. This question was previously considered in the case of fission with respect to the quadrupole $\left(q_{20}\right)$ and hexadecapole $\left(q_{40}\right)$ collective degrees of freedom by Berger et al. [2, 6]. We present here a more general approach to the calculation of the dissipation energy and apply it for the first time to the quadrupole and octupole collective coordinates. Although we illustrate the method for the most likely fission in ${ }^{240} \mathrm{Pu}$, it can readily be applied to any fission mode.

The problem is illustrated in Fig. 1 which shows a schematic fission saddle as a function of generic coordinates $x$ and $y$. The coordinate $y$ represents the "longitudinal" degree of freedom of the nucleus that varies as the system moves toward scission (e.g., the separation distance between pre-fragments), and $x$ represents the remaining transverse degrees of freedom. In a one-dimensional problem, where transverse motion in the $x$ coordinate is not allowed, we expect the full energy available at the exit point to the right of the plot, $\Delta E=E_{1 \mathrm{D}}-V$, will be converted to pre-scission kinetic energy in the $y$ coordinate.

Any motion in the transverse $x$ coordinate can take away from the pre-scission kinetic energy and will appear as excitation energy of the fragments after scission. Therefore the amount of energy dissipated into the transverse degree of freedom in Fig. 1 can be deduced by comparing calculations in 1D to those in higher dimensions. The central concept we demonstrate and take advantage of in the present work is that if the probability flux of the wave function representing the fissioning nucleus, normalized by the squared amplitude of that wave function is constant in time at some "exit" (or fission) point, then the dissipated energy can be obtained from an analysis of the flux at that point alone, without the need to examine the wave function over the remaining full set of collective coordinates. This powerful result, which follows from the WKB approximation, leads to a tremendous simplification of the problem. The object of the present paper, therefore, is to first identify the longitudinal and transverse coordinates for a fissioning nucleus described by its quadrupole $\left(q_{20}\right)$ and octupole $\left(q_{30}\right)$ moments, and to then deduce the energy dissipated in the transverse coordinate by comparing 1D and 2D calculations at the exit point. 


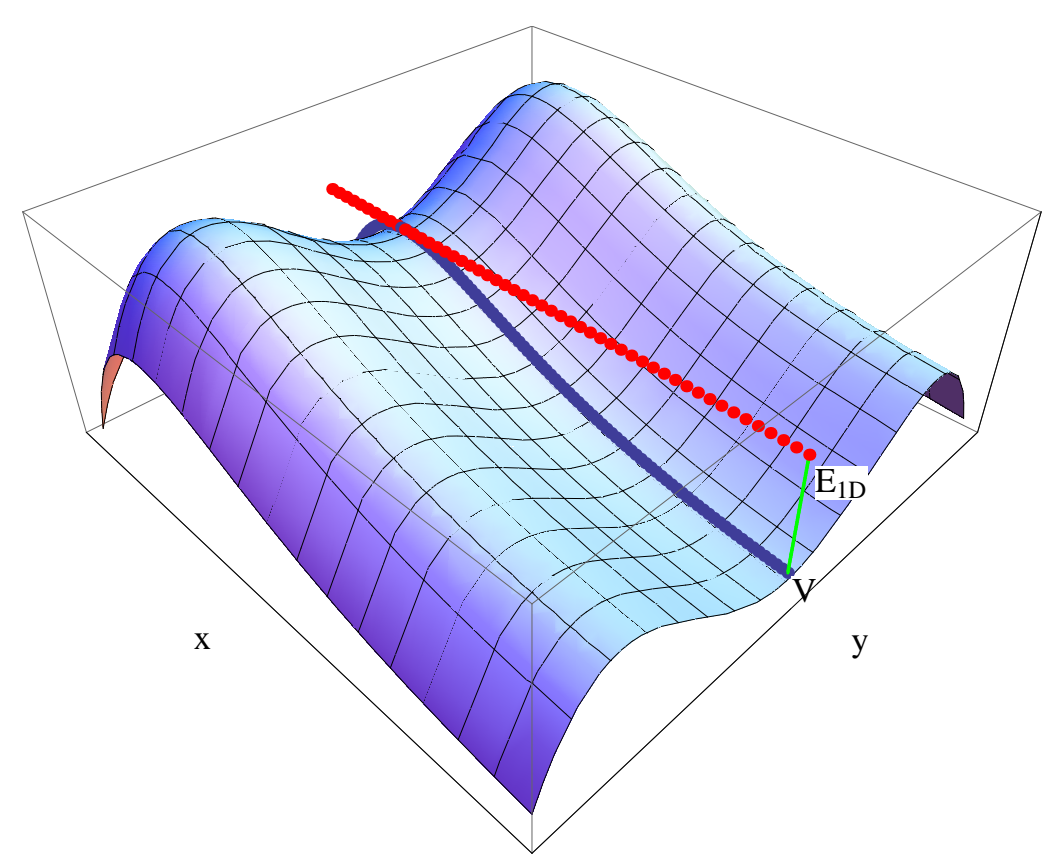

Figure 1: Schematic potential energy surface for fission with respect to longitudinal $(x)$ and transverse $(y)$ coordinates. An amount of energy $E_{1 \mathrm{D}}-V$, to be partitioned between kinetic and excitation energies of the fragments, is made available in the descent from saddle to scission.

\section{THEORY}

The derivation of the necessary formalism is broken down into several steps below. In section II A we determine the direction in the $\left(q_{20}, q_{30}\right)$ plane which gives a maximum probability flux at an exit point of interest. We take the maximum-flux direction in the $\left(q_{20}, q_{30}\right)$ plane as the longitudinal fission direction. In section II B we transform the collective Schrödinger equation from $\left(q_{20}, q_{30}\right)$ to the new set of fission coordinates $(x, y)$ aligned with the longitudinal and transverse fission directions. In section II C, we calculate a normalized flux in the longitudinal fission direction. Finally, 1D and 2D calculations of the normalized flux are combined in section IID, and the expression for the energy dissipated into transverse collective modes is derived.

\section{A. Calculation of the maximum probability flux}

Starting from the continuity equation (Eq. (A3) derived in appendix A),

$$
\frac{\partial}{\partial t} \iint_{\mathcal{V}} d q_{20} d q_{30} \Psi^{*} \Psi=-\iint_{\mathcal{V}} d q_{20} d q_{30} \nabla \cdot \vec{j}
$$


we note that the integral on the right-hand side (excluding the minus sign) is the probability flux leaving region $\mathcal{V}$. This integral can be further expanded,

$$
\iint_{\mathcal{V}} d q_{20} d q_{30} \nabla \cdot \vec{j}=\iint_{\mathcal{V}} d q_{20} d q_{30}\left(\frac{\partial j_{2}}{\partial q_{20}}+\frac{\partial j_{3}}{\partial q_{30}}\right)
$$

For a fixed $q_{30}$ we integrate $q_{20}$ between the limits of the contour $\mathcal{S}$ enclosing region $\mathcal{V}$ and likewise for fixed $q_{20}$ we integrate between the limits in $q_{30}$, which yields

$$
\begin{aligned}
\iint_{\mathcal{V}} d q_{20} d q_{30}\left(\frac{\partial j_{2}}{\partial q_{20}}+\frac{\partial j_{3}}{\partial q_{30}}\right)= & \int d q_{30}\left[j_{2}\left(q_{20}^{(\max )}, q_{30}\right)-j_{2}\left(q_{20}^{(\min )}, q_{30}\right)\right]+ \\
& \int d q_{20}\left[j_{3}\left(q_{20}, q_{30}^{(\max )}\right)-j_{3}\left(q_{20}, q_{30}^{(\min )}\right)\right]
\end{aligned}
$$

and where the points $\left(q_{20}^{(\max )}, q_{30}\right),\left(q_{20}^{(\min )}, q_{30}\right),\left(q_{20}, q_{30}^{(\max )}\right)$, and $\left(q_{20}, q_{30}^{(\min )}\right)$ are all located on the contour $\mathcal{S}$. By construction, we choose region $\mathcal{V}$ such that the current vanishes at the lower bound of the contour, and we are therefore left with

$$
\iint_{\mathcal{V}} d q_{20} d q_{30} \nabla \cdot \vec{j}=\int d q_{30} j_{2}\left(q_{20}^{(\max )}, q_{30}\right)+\int d q_{20} j_{3}\left(q_{20}, q_{30}^{(\max )}\right)
$$

We focus on a small segment $\delta \mathcal{S}$ along the contour $\mathcal{S}$ and calculate the flux $\delta \Phi$ through that segment. Using Eq. (1) and assuming the segment is small enough that the current is constant all along its length,

$$
\delta \Phi=j_{3} \delta q_{20}-j_{2} \delta q_{30}
$$

The minus sign in this expression is due to the geometry of the problem, shown in Fig. 2 , which requires the segment $\delta \mathcal{S}$ to have a negative slope (and therefore $\delta q_{30}<0$ ) for a positive flux in the $q_{20}$ direction.

Given an initial segment with slope $m \equiv \delta q_{30} / \delta q_{20}$, we wish to rotate that segment to find the orientation that yields the maximum flux. In order to perform this rotation, we must work in a dimensionless coordinate system. Therefore, we introduce scaling factors $\Delta q_{20}$ and $\Delta q_{30}$ and choose them so as to preserve the value of the slope of segment $\delta \mathcal{S}$,

$$
m=\frac{\delta q_{30} / \Delta q_{30}}{\delta q_{20} / \Delta q_{20}}
$$

in other words, although the units of $\Delta q_{20}$ and $\Delta q_{30}$ are different, their numerical values must remain the same, and we choose them to be $\Delta q_{20}=1 \mathrm{~b}$ and $\Delta q_{30}=1 \mathrm{~b}^{3 / 2}$, without 


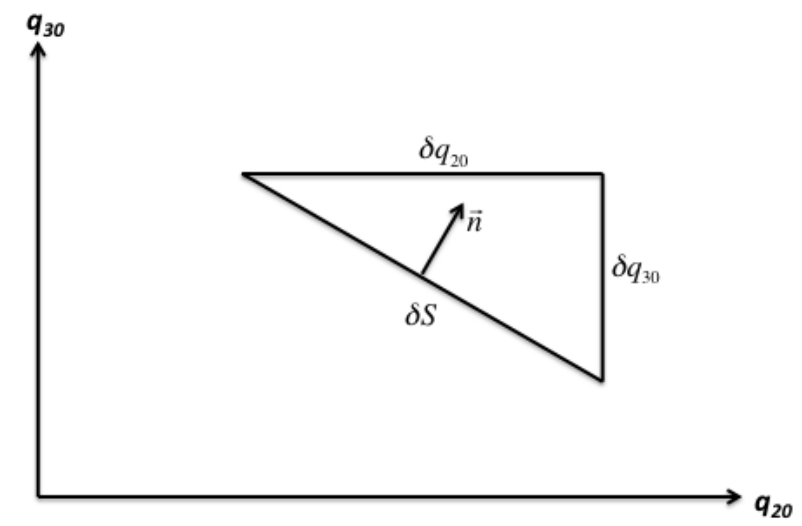

Figure 2: Geometry of the segment $\delta \mathcal{S}$ in the $\left(q_{20}, q_{30}\right)$ coordinate system (see text for explanation). loss of generality. We can then write

$$
\begin{aligned}
\frac{\delta q_{20}}{\Delta q_{20}} & \equiv \varepsilon \cos \theta \\
\frac{\delta q_{30}}{\Delta q_{30}} & \equiv \varepsilon \sin \theta
\end{aligned}
$$

where $\varepsilon$ is dimensionless. We show in section IID that the final result, the calculated dissipation energy, does not depend on the value of $\varepsilon$. The flux in Eq. (2) is now

$$
\delta \Phi=\varepsilon\left(j_{3} \Delta q_{20} \cos \theta-j_{2} \Delta q_{30} \sin \theta\right)
$$

and is maximized when

$$
\tan \theta_{\max }=-\frac{j_{2} \Delta q_{30}}{j_{3} \Delta q_{20}}
$$

In the remainder of this report, we will take this angle as the longitudinal direction of fission. For this angle, we find the maximum positive flux

$$
\delta \Phi_{\max }=\varepsilon \sqrt{\left(j_{3} \Delta q_{20}\right)^{2}+\left(j_{2} \Delta q_{30}\right)^{2}}
$$

With the angle $\theta_{\max }$ given in Eq. (5), we have thus defined a new set of coordinates $(x, y)$, with the $x$ axis taken along the direction of maximum flux at each $\left(q_{20}, q_{30}\right)$ point (which we 
interpret as the longitudinal fission coordinate), and the $y$ axis (representing the transverse fission coordinate) perpendicular to it. The coordinate transformation from $\left(q_{20}, q_{30}\right)$ to $(x, y)$ is derived in appendix , and consists of a scaling and a rotation given by Eq. (B1),

$$
\left(\begin{array}{l}
x \\
y
\end{array}\right)=\left(\begin{array}{c}
-\frac{\sin \theta_{\max }}{\varepsilon \Delta q_{20}} q_{20}+\frac{\cos \theta_{\max }}{\varepsilon \Delta q_{30}} q_{30} \\
-\frac{\cos \theta_{\max }}{\varepsilon \Delta q_{20}} q_{20}-\frac{\sin \theta_{\max }}{\varepsilon \Delta q_{30}} q_{30}
\end{array}\right)
$$

\section{B. The Schrödinger equation in the fission coordinates}

Starting from the Schrödinger equation in the $\left(q_{20}, q_{30}\right)$ coordinates,

$$
\left[-\frac{1}{2} \sum_{i, j=2,3} \frac{\partial}{\partial q_{i, 0}} B_{i, j} \frac{\partial}{\partial q_{j, 0}}+V\left(q_{20}, q_{30}\right)\right] \Psi\left(q_{20}, q_{30}, t\right)=\hbar i \frac{\partial}{\partial t} \Psi\left(q_{20}, q_{30}, t\right)
$$

we re-write this equation in the $(x, y)$ coordinates defined by Eq. (B1). First, we calculate the Jacobian of the transformation

$$
\begin{aligned}
J & \equiv\left|\frac{\partial\left(q_{20}, q_{30}\right)}{\partial(x, y)}\right| \\
& =\varepsilon^{2} \Delta q_{20} \Delta q_{30}
\end{aligned}
$$

and introduce the normalized wave function solution $g(x, y, t)$ where

$$
\Psi\left(q_{20}, q_{30}, t\right) \equiv J^{-1 / 2} g(x, y, t)
$$

Next, we relate $B_{\alpha \beta}(x, y)$, where $\alpha$ and $\beta$ stand for either of the new coordinates $x$ and $y$, to $B_{i, j}\left(q_{20}, q_{30}\right)$ by

$$
B_{i j}\left(q_{20}, q_{30}\right) \equiv \sum_{\alpha, \beta=x, y} B_{\alpha \beta}(x, y) \frac{\partial q_{i, 0}}{\partial \alpha} \frac{\partial q_{j, 0}}{\partial \beta}
$$

Then,

$$
\begin{aligned}
\sum_{i, j} \frac{\partial}{\partial q_{i, 0}} B_{i, j} \frac{\partial}{\partial q_{j, 0}} & =\sum_{i, j}\left(\sum_{\alpha^{\prime}} \frac{\partial \alpha^{\prime}}{\partial q_{i, 0}} \frac{\partial}{\partial \alpha^{\prime}}\right) \sum_{\alpha, \beta} B_{\alpha \beta} \frac{\partial q_{i, 0}}{\partial \alpha} \frac{\partial q_{j, 0}}{\partial \beta}\left(\sum_{\beta^{\prime}} \frac{\partial \beta^{\prime}}{\partial q_{j, 0}} \frac{\partial}{\partial \beta^{\prime}}\right) \\
& =\sum_{\alpha, \beta} \sum_{\alpha^{\prime}, \beta^{\prime}}\left(\sum_{i} \frac{\partial \alpha^{\prime}}{\partial q_{i, 0}} \frac{\partial q_{i, 0}}{\partial \alpha}\right) \frac{\partial}{\partial \alpha^{\prime}} B_{\alpha \beta}\left(\sum_{j} \frac{\partial \beta^{\prime}}{\partial q_{j, 0}} \frac{\partial q_{j, 0}}{\partial \beta}\right) \frac{\partial}{\partial \beta^{\prime}} \\
& =\sum_{\alpha, \beta} \frac{\partial}{\partial \alpha} B_{\alpha \beta} \frac{\partial}{\partial \beta}
\end{aligned}
$$


and q. (6) becomes

$$
\left[-\frac{1}{2} \sum_{\alpha, \beta=x, y} \frac{\partial}{\partial \alpha} B_{\alpha \beta} \frac{\partial}{\partial \beta}+V(x, y)\right] g(x, y, t)=\hbar i \frac{\partial}{\partial t} g(x, y, t)
$$

with $V(x, y)=V\left(x\left(q_{20}, q_{30}\right), y\left(q_{20}, q_{30}\right)\right)$. In closing, we note that Eq. (7) can be inverted,

$$
B_{\alpha \beta}(x, y)=\sum_{i, j=2,3} B_{i j}\left(q_{20}, q_{30}\right) \frac{\partial \alpha}{\partial q_{i, 0}} \frac{\partial \beta}{\partial q_{j, 0}}
$$

from which we can calculate the inertia tensor in the new coordinates $(x, y)$. In particular, we will need

$$
B_{x x}=B_{22}\left(\frac{-\sin \theta}{\varepsilon \Delta q_{20}}\right)^{2}-2 B_{23} \frac{\cos \theta \sin \theta}{\varepsilon^{2} \Delta q_{20} \Delta q_{30}}+B_{33}\left(\frac{\cos \theta}{\varepsilon \Delta q_{30}}\right)^{2}
$$

\section{The normalized flux in the longitudinal fission direction}

The current in the $(x, y)$ fission coordinates is derived in appendix C. We now wish to calculate the flux through a line segment in the $(x, y)$ plane corresponding to the probability current $\vec{j}$. We follow the same approach used in section II A, but this time for a segment with slope $\Delta y / \Delta x$. The equation of the supporting line is

$$
f(x, y)=\Delta x\left(y-y_{0}\right)-\Delta y\left(x-x_{0}\right)=0
$$

where $\left(x_{0}, y_{0}\right)$ is a point on that line (e.g., the point $A$ ). A normal vector to that line is then given by

$$
\begin{aligned}
\vec{n} & =\nabla f \\
& =\left(\begin{array}{c}
-\Delta y \\
\Delta x
\end{array}\right)
\end{aligned}
$$

and its norm is

$$
|\vec{n}|=\sqrt{\Delta x^{2}+\Delta y^{2}}
$$

The flux through the line segment is then given by

$$
\Delta \Phi=\vec{j} \cdot \frac{\vec{n}}{|\vec{n}|} \Delta s
$$


where $\Delta s=\sqrt{\Delta x^{2}+\Delta y^{2}}$ is the length of the segment. Thus, we have

$$
\Delta \Phi=-j_{x} \Delta y+j_{y} \Delta x
$$

Using Eq. (D1), we write the components of the current explicitly,

$$
\begin{aligned}
& j_{x}=\varepsilon \Delta q_{20} \Delta q_{30}\left[-\frac{\sin \theta}{\Delta q_{20}} j_{2}+\frac{\cos \theta}{\Delta q_{30}} j_{3}\right] \\
& j_{y}=\varepsilon \Delta q_{20} \Delta q_{30}\left[-\frac{\cos \theta}{\Delta q_{20}} j_{2}-\frac{\sin \theta}{\Delta q_{30}} j_{3}\right]
\end{aligned}
$$

If we now choose $\theta$ to be the angle for which the flux is maximized, given by Eq. (5), then we have

$$
j_{y}=0
$$

so that the direction of maximum flux is entirely along the $x$ axis, as expected. In that case,

$$
j_{x}= \pm \varepsilon \Delta q_{20} \Delta q_{30} \sqrt{\left(\frac{j_{2}}{\Delta q_{20}}\right)^{2}+\left(\frac{j_{3}}{\Delta q_{30}}\right)^{2}}
$$

Then, the (positive) flux is given by

$$
\Delta \Phi=\Delta y \varepsilon \Delta q_{20} \Delta q_{30} \sqrt{\left(\frac{j_{2}}{\Delta q_{20}}\right)^{2}+\left(\frac{j_{3}}{\Delta q_{30}}\right)^{2}}
$$

Since we are only interested in a specific division of the nucleus (the most likely fission mode in the present work) at a given exit point, rather than the entire set of possible fragments, the flux $\Delta \Phi$ can vary significantly over time. A more stable quantity, as we will see in section II D, can be obtained by normalizing the flux by the squared amplitude of the collective wave function. We can calculate this normalized flux,

$$
\Delta \Phi_{N} \equiv \frac{\Delta \Phi}{\int d y|g(x, y, t)|^{2}}
$$

where the integral in the denominator is carried out over the segment length $\Delta y$. For a length $\Delta y$ sufficiently small that $g(x, y, t)$ can be considered constant, we have

$$
\Delta \Phi_{N}=\frac{1}{\varepsilon\left|\Psi\left(q_{20}, q_{30}, t\right)\right|^{2}} \sqrt{\left(\frac{j_{2}}{\Delta q_{20}}\right)^{2}+\left(\frac{j_{3}}{\Delta q_{30}}\right)^{2}}
$$




\section{Flux in the WKB approximation}

Suppose the inertia and potential in Eq. (8) are essentially constant with respect to $x$ and $y$, and that the motion is entirely in the $x$ direction. The Schrödinger equation then reduces to

$$
\left[-\frac{1}{2} B_{x x} \frac{\partial^{2}}{\partial x^{2}}+V\right] g(x, t)=\hbar i \frac{\partial}{\partial t} g(x, t)
$$

and the solution is the plane wave

$$
g(x, t)=\mathcal{N} \exp \left[i\left(k_{x} x-\frac{E_{1 \mathrm{D}}}{\hbar} t\right)\right]
$$

where $\mathcal{N}$ is a normalization constant, $k_{x}$ is the wave number, and $E_{1 \mathrm{D}}$ is the energy of the solution. Inserting Eq. (12) into Eq. (11) leads to the relation

$$
\frac{1}{2} B_{x} k_{x}^{2}+V=E_{1 \mathrm{D}}
$$

or

$$
k_{x}=\sqrt{\frac{2}{B_{x x}}\left(E_{1 \mathrm{D}}-V\right)}
$$

The probability current in this case is

$$
\begin{aligned}
j_{x} & =\frac{1}{2 \hbar i} B_{x x}\left(g^{*} \frac{\partial}{\partial y} g-g \frac{\partial}{\partial y} g^{*}\right) \\
& =\frac{B_{x x}}{\hbar} \mathcal{N}^{2} k_{x}
\end{aligned}
$$

and the normalized flux is

$$
\begin{aligned}
\Delta \Phi_{N}^{(1 \mathrm{D})} & =\frac{j_{x} \Delta y}{|g(x, y, t)|^{2} \Delta y} \\
& =\frac{B_{x x}}{\hbar} k_{x} \\
& =\frac{1}{\hbar} \sqrt{2 B_{x x}\left(E_{1 \mathrm{D}}-V\right)}
\end{aligned}
$$

where the inertia $B_{x x}$ is given explicitly by Eq. (9). Note that the normalized flux $\Delta \Phi_{N}^{(1 \mathrm{D})}$ does not depend on the value of $\mathcal{N}$. This is a very important property, because it shows that if the WKB approximation is valid, then the normalized flux is insensitive to fluctuations and changes in the wave function. 
In order to determine the amount of dissipated energy, we interpret the flux $\Delta \Phi_{N}$ given by Eq. (10) using the 1D formula in Eq. (13). Unlike the 1D case however, we do not expect the full available energy $E_{1 \mathrm{D}}-V$ to be converted to kinetic energy, but rather only a portion $E_{2 \mathrm{D}}-V$, with the difference dissipated into the transverse degrees of freedom. Then the ratio of normalized fluxes is

$$
\frac{\Delta \Phi_{N}}{\Delta \Phi_{N}^{(1 \mathrm{D})}}=\sqrt{\frac{E_{2 \mathrm{D}}-V}{E_{1 \mathrm{D}}-V}}
$$

from which we deduce the energy in the $2 \mathrm{D}$ case

$$
E_{2 \mathrm{D}}=V+\left(\frac{\Delta \Phi_{N}}{\Delta \Phi_{N}^{(1 \mathrm{D})}}\right)^{2}\left(E_{1 \mathrm{D}}-V\right)
$$

and the dissipated energy is

$$
\Delta E_{\text {coll }} \equiv E_{1 \mathrm{D}}-E_{2 \mathrm{D}}
$$

This dissipated energy is the quantity of interest we calculate in this paper. Note that, because of Eq. (9), $B_{x x} \propto \varepsilon^{-2}$ and therefore $\Delta \Phi_{N}^{(1 \mathrm{D})} \propto \varepsilon^{-1}$ and furthermore, by Eq. (10), $\Delta \Phi_{N} \propto \varepsilon^{-1}$ as well. Therefore the result in Eq. (15), which follows from the ratio of fluxes in Eq. (14), does not depend on $\varepsilon$.

\section{RESULTS}

We have calculated the energy dissipated into the transverse collective degrees for ${ }^{240} \mathrm{Pu}$ using the approach described above. The time-dependent calculation of fission for this nucleus was performed using the approach outlined in [4]. In order to calculate the 2D flux, we have solved the equations of a Time-Dependent Generator-Coordinate Method (TDGCM) in the Bohr approximation [5]. An initial state just above the first barrier and with energy $E_{1 \mathrm{D}}=-1791.9 \mathrm{MeV}$ was evolved to scission, and the calculations were carried out to a time $t=6.1 \mathrm{zs}[9]$, when only $\approx 1 \%$ of the initial wave packet has still not fissioned. The analysis of the resulting flux was performed at the exit point $\left(q_{20}, q_{30}\right)=\left(368 \mathrm{~b}, 60 \mathrm{~b}^{3 / 2}\right)$, the last point along the fission valley before scission occurs. In the vicinity of this exit point, the potential energy and inertia tensor are fairly constant (within a few \%), and the WKB approximation used in section II D is therefore justified. 


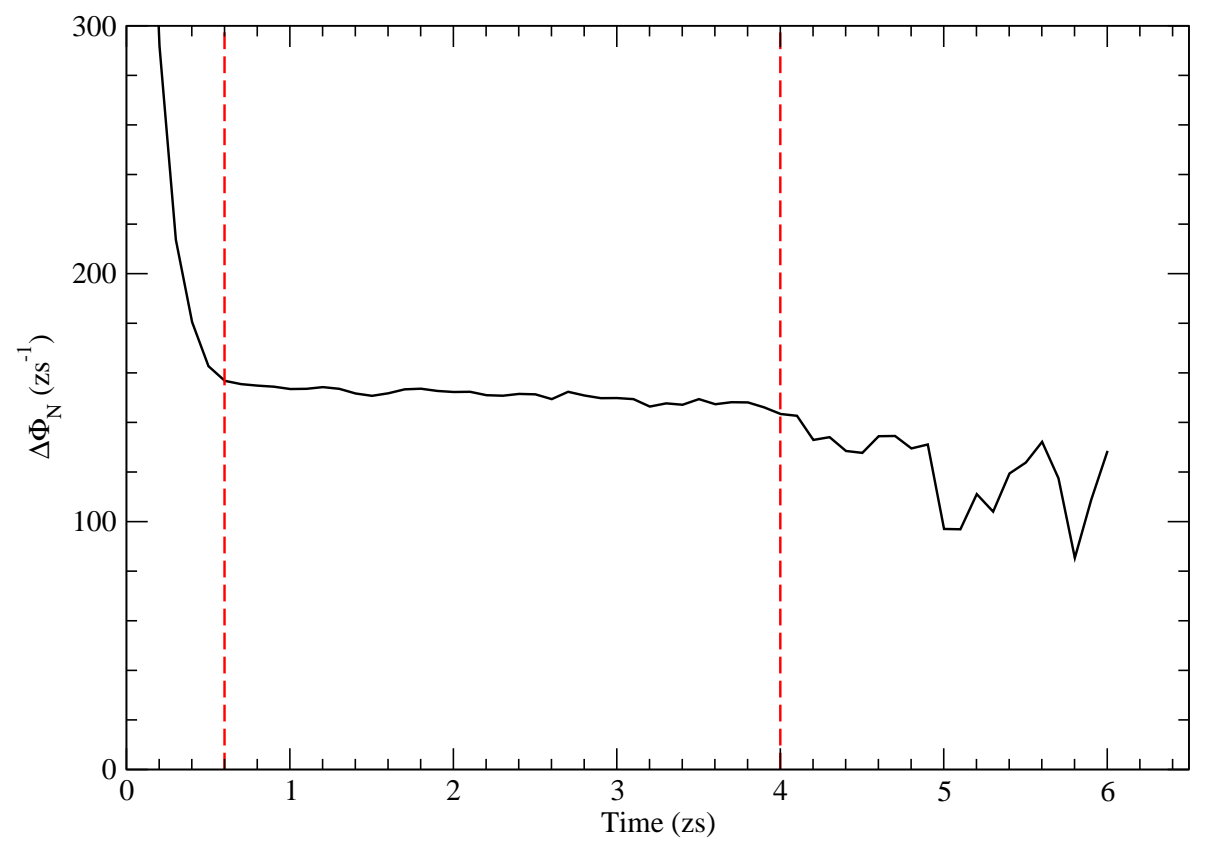

Figure 3: Normalized flux at the exit point $\left(q_{20}, q_{30}\right)=\left(368 \mathrm{~b}, 60 \mathrm{~b}^{3 / 2}\right)$, plotted as a function of time. The vertical dashed lines delimit a region ( $0.6 \mathrm{zs} \leq t \leq 4.0 \mathrm{zs})$ where the normalized flux is relatively constant in time, and can therefore be used in the analysis of the energy dissipation.

The normalized flux $\Delta \Phi_{N}$ calculated with Eq. (10) is plotted in Fig. 3 as a function of time. The flux shows significant fluctuations at early times $(t \lesssim 0.6 \mathrm{zs})$ before a sufficiently large fraction of the wave packet has reached the exit point, and at late times $(t \gtrsim 4.0 \mathrm{zs})$ when nearly all the wave packet has passed the exit points and only a trickle (about 3.6\%) is left to contribute to the flux. In between those times, however, the normalized flux is remarkably constant. It is this constant behavior which justifies, a posteriori, the WKB approximation we use in our interpretation (Eq. (13)). Note that the fission times obtained in this calculation, $\approx 4 \times 10^{-21} \mathrm{~s}$, are essentially identical to those found by Berger et al. [6].

Combining the data for those times $(0.6 \mathrm{zs} \leq t \leq 4.50 \mathrm{zs})$ when the normalized flux is constant, we extract 35 individual estimates, one for each time step of $0.1 \mathrm{zs}$, of the dissipation energy using Eq. (15). The mean and standard deviation of these 35 values are 
3.4 and 0.5 , respectively, from which we deduce an average collective dissipation energy

$$
\overline{\Delta E}=3.4 \pm 0.1
$$

where the uncertainty is the standard deviation of the sample mean. This is the energy of the descent from saddle to scission in the $\left(q_{20}, q_{30}\right)$ coordinates that would appear as excitation energy of the fragments.

\section{CONCLUSION}

We have calculated a value of $3.4 \pm 0.1 \mathrm{MeV}$ for the energy dissipated in the transverse collective degree of freedom for ${ }^{240} \mathrm{Pu}$ fission in the $\left(q_{20}, q_{30}\right)$ plane. This value was obtained by comparing the normalized flux of the collective wave packet in one and two dimensions. This result is similar to the $2.1 \mathrm{MeV}$ dissipation calculated by Berger et al. [6] in the $\left(q_{20}, q_{40}\right)$ plane. The dissipation energy calculated in the present work, combined with that calculated by Berger et al. supports the conjecture that each additional collective degree of freedom in fission contributes $\sim 2 \mathrm{MeV}$ to the dissipated energy. The calculations presented here could be improved in the future by expanding the analysis to higher dimensions, e.g. comparing TDGCM calculations in the $\left(q_{20}, q_{30}, q_{40}\right)$ plane to the one-dimensional case. Eventually, multi-dimensional calculations including the coupling with intrinsic excitations could provide an accurate estimate of the entire energy dissipated in all relevant transverse degrees of freedom.

\section{Appendix A: Continuity equation in the $\left(q_{20}, q_{30}\right)$ coordinates}

Consider the collective wave function $\Psi\left(q_{20}, q_{30}, t\right)$. We wish to calculate the corresponding probability flux through a given length of arc. We begin with the Schrödinger representation of the wave function

$$
\Psi\left(q_{20}, q_{30}, t\right)=e^{-i H t / \hbar} \Psi\left(q_{20}, q_{30}, 0\right)
$$

from which we obtain the standard time-dependent Schrödinger equation

$$
\hbar i \frac{\partial}{\partial t} \Psi\left(q_{20}, q_{30}, t\right)=H \Psi\left(q_{20}, q_{30}, t\right)
$$


and its complex conjugate is

$$
-\hbar i \frac{\partial}{\partial t} \Psi^{*}\left(q_{20}, q_{30}, t\right)=H \Psi^{*}\left(q_{20}, q_{30}, t\right)
$$

Combining these last two equations, we find

$$
\hbar i \frac{\partial}{\partial t} \Psi^{*} \Psi=\Psi^{*} H \Psi-\Psi H \Psi^{*}
$$

Integrating over a volume $\mathcal{V}$ in the $\left(q_{20}, q_{30}\right)$ coordinates,

$$
\hbar i \frac{\partial}{\partial t} \iint_{\mathcal{V}} d q_{20} d q_{30} \Psi^{*} \Psi=\iint_{\mathcal{V}} d q_{20} d q_{30}\left(\Psi^{*} H \Psi-\Psi H \Psi^{*}\right)
$$

On the right-hand side, we write the Hamiltonian as the sum of kinetic $T$ and potential $V$ terms, and cancel the potential term in the difference,

$$
\hbar i \frac{\partial}{\partial t} \iint_{\mathcal{V}} d q_{20} d q_{30} \Psi^{*} \Psi=\iint_{\mathcal{V}} d q_{20} d q_{30}\left(\Psi^{*} T \Psi-\Psi T \Psi^{*}\right)
$$

The kinetic energy operator is

$$
\begin{aligned}
T & =-\frac{1}{2} \sum_{i, j=2,3} \frac{\partial}{\partial q_{i, 0}} B_{i, j} \frac{\partial}{\partial q_{j, 0}} \\
& \equiv-\frac{1}{2} \nabla \cdot(B \nabla)
\end{aligned}
$$

where $B$ is the symmetric inertia tensor

$$
B=\left(\begin{array}{cc}
B_{22} & B_{23} \\
B_{23} & B_{33}
\end{array}\right)
$$

We use the following identity for the divergence

$$
\nabla \cdot(f \vec{F})=(\nabla f) \cdot \vec{F}+f \nabla \cdot \vec{F}
$$

to write

$$
\begin{aligned}
\Psi^{*} T \Psi-\Psi T \Psi^{*}= & -\frac{1}{2}\left[\nabla \cdot\left(\Psi^{*} B \nabla \Psi\right)-\left(\nabla \Psi^{*}\right) \cdot(B \nabla \Psi)\right]+ \\
& \frac{1}{2}\left[\nabla \cdot\left(\Psi B \nabla \Psi^{*}\right)-(\nabla \Psi) \cdot\left(B \nabla \Psi^{*}\right)\right]
\end{aligned}
$$

we can check that the difference

$$
\left(\nabla \Psi^{*}\right) \cdot(B \nabla \Psi)-(\nabla \Psi) \cdot\left(B \nabla \Psi^{*}\right)=0
$$


by explicit substitution of $B$, and we are therefore left with

$$
\Psi^{*} T \Psi-\Psi T \Psi^{*}=-\frac{1}{2} \nabla \cdot\left(\Psi^{*} B \nabla \Psi-\Psi B \nabla \Psi^{*}\right)
$$

Next, we define the probability current

$$
\vec{j}=\frac{1}{2 \hbar i} B\left(\Psi^{*} \nabla \Psi-\Psi \nabla \Psi^{*}\right)
$$

and return to Eqs. (A1) and (A2) to write the continuity equation in its integral form,

$$
\frac{\partial}{\partial t} \iint_{\mathcal{V}} d q_{20} d q_{30} \Psi^{*} \Psi=-\iint_{\mathcal{V}} d q_{20} d q_{30} \nabla \cdot \vec{j}
$$

\section{Appendix B: Change of coordinates}

We now derive explicitly the transformation to the system of coordinates with axes along the direction of maximum flux and perpendicular to it defined by the angle $\theta_{\max }$ in the previous section. We begin with the transformation of $q_{20}$ and $q_{30}$ to dimensionless coordinates using the scaling factors $\Delta q_{20}$ and $\Delta q_{30}$ in Eq. (4),

$$
\begin{aligned}
u & \equiv \frac{q_{20}}{\Delta q_{20}} \\
v & \equiv \frac{q_{30}}{\Delta q_{30}}
\end{aligned}
$$

Then, the equation of the line going through a given point $\left(q_{20}^{(0)}, q_{30}^{(0)}\right)$ and with slope $\delta q_{30} / \delta q_{20}$ is

$$
\delta q_{20}\left(q_{30}-q_{30}^{(0)}\right)-\delta q_{30}\left(q_{20}-q_{20}^{(0)}\right)=0
$$

or, in the dimensionless coordinates $(u, v)$,

$$
\varepsilon \cos \theta\left(v-v_{0}\right)-\varepsilon \sin \theta\left(u-u_{0}\right)=0
$$

The left-hand side of this equation defines a function

$$
f(u, v) \equiv \varepsilon \cos \theta\left(v-v_{0}\right)-\varepsilon \sin \theta\left(u-u_{0}\right)
$$

whose gradient gives a vector normal to the line is given by

$$
\begin{aligned}
\vec{n} & =\nabla f \\
& =\left(\begin{array}{c}
-\varepsilon \sin \theta \\
\varepsilon \cos \theta
\end{array}\right)
\end{aligned}
$$


and with a tangent vector

$$
\vec{t}=\left(\begin{array}{c}
-\varepsilon \cos \theta \\
-\varepsilon \sin \theta
\end{array}\right)
$$

where the sign is chosen so that for $\theta=-\pi / 2$, the vector $\vec{t}$ is in the direction of the positive $q_{30}$ axis. We now wish to find the transformation $A$ to the system of coordinates with $x$ along the longitudinal fission direction and $y$ along the transverse direction. In other words,

$$
A \vec{n}=\left(\begin{array}{l}
1 \\
0
\end{array}\right)
$$

and

$$
A \vec{t}=\left(\begin{array}{l}
0 \\
1
\end{array}\right)
$$

The solution to the resulting system of equations is

$$
A=\frac{1}{\varepsilon}\left(\begin{array}{cc}
-\sin \theta & \cos \theta \\
-\cos \theta & -\sin \theta
\end{array}\right)
$$

The new set of coordinates $(x, y)$ is therefore related to the coordinates $\left(q_{20}, q_{30}\right)$ by

$$
\begin{aligned}
\left(\begin{array}{l}
x \\
y
\end{array}\right) & =\frac{1}{\varepsilon}\left(\begin{array}{cc}
-\sin \theta & \cos \theta \\
-\cos \theta & -\sin \theta
\end{array}\right)\left(\begin{array}{l}
q_{20} / \Delta q_{20} \\
q_{30} / \Delta q_{30}
\end{array}\right) \\
& =\left(\begin{array}{c}
-\frac{\sin \theta}{\varepsilon \Delta q_{20}} q_{20}+\frac{\cos \theta}{\varepsilon \Delta q_{30}} q_{30} \\
-\frac{\cos \theta}{\varepsilon \Delta q_{20}} q_{20}-\frac{\sin \theta}{\varepsilon \Delta q_{30}} q_{30}
\end{array}\right)
\end{aligned}
$$

\section{Appendix C: Continuity equation in the fission coordinates}

Next, we calculate the probability current from Eq. (8) and its complex conjugate in the standard way,

$$
-\frac{1}{2}\left[g^{*}\left(\sum_{\alpha, \beta} \frac{\partial}{\partial \alpha} B_{\alpha \beta} \frac{\partial}{\partial \beta}\right) g-g\left(\sum_{\alpha, \beta} \frac{\partial}{\partial \alpha} B_{\alpha \beta} \frac{\partial}{\partial \beta}\right) g^{*}\right]=\hbar i \frac{\partial}{\partial t} g^{*} g
$$

Noting that

$$
\frac{\partial}{\partial \alpha} g^{*} B_{\alpha \beta} \frac{\partial}{\partial \beta} g=\left(\frac{\partial g^{*}}{\partial \alpha}\right) B_{\alpha \beta}\left(\frac{\partial g}{\partial \beta}\right)+g^{*} \frac{\partial}{\partial \alpha} B_{\alpha \beta} \frac{\partial}{\partial \beta} g
$$


and, similarly,

$$
\frac{\partial}{\partial \alpha} g B_{\alpha \beta} \frac{\partial}{\partial \beta} g^{*}=\left(\frac{\partial g}{\partial \alpha}\right) B_{\alpha \beta}\left(\frac{\partial g^{*}}{\partial \beta}\right)+g \frac{\partial}{\partial \alpha} B_{\alpha \beta} \frac{\partial}{\partial \beta} g^{*}
$$

so that

$$
g^{*} \frac{\partial}{\partial \alpha} B_{\alpha \beta} \frac{\partial}{\partial \beta} g-g \frac{\partial}{\partial \alpha} B_{\alpha \beta} \frac{\partial}{\partial \beta} g^{*}=\frac{\partial}{\partial \alpha} B_{\alpha \beta}\left(g^{*} \frac{\partial g}{\partial \beta}-g \frac{\partial g^{*}}{\partial \beta}\right)
$$

an then we can rewrite Eq. (C1) as

$$
-\frac{1}{2 \hbar i} \sum_{\alpha} \frac{\partial}{\partial \alpha} \sum_{\beta} B_{\alpha \beta}\left(g^{*} \frac{\partial g}{\partial \beta}-g \frac{\partial g^{*}}{\partial \beta}\right)=\frac{\partial}{\partial t} g^{*} g
$$

We define the probability current $\vec{j}$ with components

$$
j_{\alpha} \equiv \frac{1}{2 \hbar i} \sum_{\beta} B_{\alpha \beta}\left(g^{*} \frac{\partial g}{\partial \beta}-g \frac{\partial g^{*}}{\partial \beta}\right)
$$

so that Eq. (C2) becomes the well-known continuity equation

$$
\nabla \cdot \vec{j}+\frac{\partial \rho}{\partial t}=0
$$

where

$$
\rho(x, y, t) \equiv g^{*}(x, y, t) g(x, y, t)
$$

\section{Appendix D: Coordinate transformation of the probability current}

Consider the probability current in coordinates $(x, y)$ and with components given by Eq. (C3),

$$
j_{\alpha} \equiv \frac{1}{2 \hbar i} \sum_{\beta} B_{\alpha \beta}\left(g^{*} \frac{\partial g}{\partial \beta}-g \frac{\partial g^{*}}{\partial \beta}\right)
$$

the corresponding current in the $\left(q_{20}, q_{30}\right)$ coordinates has components

$$
j_{m} \equiv \frac{1}{2 \hbar i} \sum_{n} B_{m n}\left(\Psi^{*} \frac{\partial \Psi}{\partial q_{n, 0}}-\Psi \frac{\partial \Psi^{*}}{\partial q_{n, 0}}\right)
$$

and we wish to find a relation connecting these components in the two different coordinate systems. For this, we calculate the quantity

$$
\begin{aligned}
\sum_{m} \frac{\partial \alpha}{\partial q_{m, 0}} j_{m} & =\frac{1}{2 \hbar i} \sum_{m, n} B_{m n} \frac{\partial \alpha}{\partial q_{m, 0}} \sum_{\beta} \frac{\partial \beta}{\partial q_{n, 0}}\left(\Psi^{*} \frac{\partial \Psi}{\partial \beta}-\Psi \frac{\partial \Psi^{*}}{\partial \beta}\right) \\
& =\frac{1}{J} \frac{1}{2 \hbar i} \sum_{\beta} B_{\alpha \beta}\left(g^{*} \frac{\partial g}{\partial \beta}-g \frac{\partial g^{*}}{\partial \beta}\right) \\
& =\frac{j_{\alpha}}{J}
\end{aligned}
$$


or,

$$
j_{\alpha}=J \sum_{m} \frac{\partial \alpha}{\partial q_{m, 0}} j_{m}
$$

[1] W. J. Swiatecki and S. Bjørnholm, Phys. Rep. 4, 325 (1972).

[2] J. F. Berger, M. Girod, and D. Gogny, Comp. Phys. Comm. 63, 365 (1991).

[3] W. Younes and D. Gogny, Phys. Rev. C 80, 054313 (2009).

[4] H. Goutte, J.-F. Berger, P. Casoli, and D. Gogny, Phys. Rev. C 71, 024316 (2005).

[5] Code "dyn0.2.f" written by H. Goutte and N. Dubray (2010).

[6] J. F. Berger, M. Girod, and D. Gogny, Nucl. Phys. A502, 85 (1989).

[7] W. Younes and D. Gogny, Phys. Rev. Lett. 107, 132501 (2011).

[8] R. Bernard, H. Goutte, D. Gogny, and W. Younes, Phys. Rev. C 84, 044308 (2011).

[9] $1 \mathrm{zs}=1$ zepto-second $=10^{-21} \mathrm{~s}$ 